\title{
Association between a point mutation at the -743-bp region of the transthyretin (TTR) gene and familial vitreous amyloidosis
}

\author{
X.M. Nie, S.J. Cai, B. Xie, X.W. Chen and M. Jiang \\ Department of Ophthalmology Affiliated Hospital of Zunyi Medical College, \\ Zui Yi, China \\ Corresponding author: S.J. Cai \\ E-mail: caishanj_ı@163.com \\ Genet. Mol. Res. 15 (1): gmr.15016926 \\ Received August 2, 2015 \\ Accepted November 13, 2015 \\ Published March 31, 2016 \\ DOI http://dx.doi.org/10.4238/gmr.15016926
}

\begin{abstract}
The aim of this study was to identify changes in the base sequence of the upstream regulatory region of the transthyretin (TTR) gene. Whole-blood DNA was extracted from ten subjects belonging to a family with familial amyloidosis vitreoretinopathy; the upstream regulatory sequence was amplified by polymerase chain reaction, detected by gel electrophoresis, and sequenced. The DNA sequence of the upstream regulatory region of the TTR gene was successfully sequenced, and a point mutation $(-743 \mathrm{~A} \rightarrow \mathrm{T})$ was identified in six of the ten blood samples: four patients and two family members without disease incidence. Therefore, a point mutation was identified in the upstream regulatory region of the TTR gene in a Han Chinese family with familial vitreous amyloidosis.
\end{abstract}

Key words: Vitreous amyloidosis; Transthyretin; Gene; Mutation; Upstream 


\section{INTRODUCTION}

Many studies have revealed that family amyloidosis is correlated with mutations in the 7616-bp transthyretin (TTR) (Westermark et al., 2002) gene located at the q11.2-q12.1 region of chromosome 18, containing four exons and three introns (Ikeda et al., 2002). So far, approximately $99 \%$ of the disease-causing mutations in the TTR gene have occurred in the second, third, and fourth exons of this gene. A previous study focusing on a Han family with vitreous amyloidosis discovered a TTR mutation site located in the third exon of chromosome 18 , inducing a mutation in the 83 rd amino acid (Gly83Arg) (Xie et al., 2013), which, however, was not expressed by several patients. Further studies have shown that the relationship between the TTR mRNA and proteins among amyloidosis patients and normal subjects was as follows: patients in a family < normal subjects in a family < healthy controls from a different family. Therefore, this disease was not exclusively characterized by the Gly83Arg mutation in exon 3 of the TTR gene. This study attempted to identify mutations in the upstream regulatory region of the TTR gene, and consequently attempted to determine the possible correlations between these mutations and amyloidosis.

\section{MATERIAL AND METHODS}

\section{Sample collection}

Whole-blood samples were obtained from 10 subjects belonging to a family with familial vitreous amyloidosis (Table 1) who visited our hospital again around the same time period; wholeblood DNA was extracted to detect the upstream regulatory region.

Table 1. Comprehensive overview of the study subjects.
\begin{tabular}{|l|l|l|c|l|l|c|c}
\hline Patient No. & No. & Gender & Age & Sickness & Mutations in third exon & Operation & Recurrence \\
\hline 1 & III-10 & Male & 42 & Yes & Gly 83 Arg & Yes & Yes \\
\hline 2 & III-7 & Female & 47 & Yes & Gly 83 Arg & Yes & Yes \\
\hline 3 & III-1 & Female & 57 & Yes & No mutation & Yes \\
\hline 4 & IV-9 & Male & 25 & Yes & No mutation & - & Yes \\
\hline 5 & III-3 & Female & 52 & Yes & Gly 83 Arg & Yes \\
\hline 6 & III-24 & Male & 43 & Yes & No mutation & No & No \\
\hline 7 & III-16 & Male & 45 & New case & No detection & Yes & No \\
\hline 8 & III-19 & Male & 38 & Yes & Gly 83 Arg & Yes & No \\
\hline 9 & III-17 & Male & 43 & Yes & Gly 83 Arg & - & - \\
\hline 10 & IV-39 & Female & 18 & No & No detection & & - \\
\hline
\end{tabular}

\section{Reagents and kits}

The Blood Genomic DNA Isolation Kit (Tiangen Biotech Co., Ltd., Beijing, China), DNA Marker (Marker V) (Tiangen Biotech Co., Ltd.), polymerase chain reaction (PCR) primers (Sangon Biotech Co., Ltd., Shanghai, China), and Resin PCR and Gel Purification Kits (SBS Genetech Co., Ltd.) were used in this study.

\section{Methods}

\section{DNA extraction and amplification by PCR}

Blood genomic DNA was extracted from the sample blood using the Blood Genomic 
DNA Isolation Kit as per the instructions provided by the manufacturer. The target exon region in the TTR gene was amplified by PCR. The upstream regulation region of the TTR gene (-1226 bp) was divided into three portions to facilitate detection. The PCR amplification was performed using the following primers (for each portion) synthesized by Invitrogen (Carlsbad, CA, USA): 663bp, F: 5'-TGGCTTCTGGTTCTCACAGTC-3', R: 5'-TGGCTTCTGGTTCTCACAGTC-3'; 623-bp, F: 5'-GTGGACTTTATCTGGGCAGAAC-3', R: 5'-TCTCCTGAGCTAGGCTGCTTAT-3'; 574-bp, F: 5'-ACGCAGTCACACAGGGAGAA-3', R: 5'-CCCAGCTCAGTAAGCTCAGTG-3'. The 25- $\mu$ L PCR system was comprised of a 10X PCR buffer $(2.5 \mu \mathrm{L}), 10 \mathrm{mM}$ dNTP mix $(0.5 \mu \mathrm{L}), 50 \mathrm{mM} \mathrm{MgCl} \mathrm{g}_{2}(0.75$ $\mu \mathrm{L}), 5 \mu \mathrm{M}$ forward primer $(1 \mu \mathrm{L}), 5 \mu \mathrm{M}$ reverse primer $(1 \mu \mathrm{L})$, DNA template $(1 \mu \mathrm{L})$, platinum Taq DNA polymerase $(0.25 \mu \mathrm{L}), \mathrm{ddH}_{2} \mathrm{O}(13 \mu \mathrm{L})$, and $5^{*} \mathrm{GC}$ buffer $(5 \mu \mathrm{L})$. The reaction conditions were as follows: initial denaturation at $94^{\circ} \mathrm{C}$ for $3 \mathrm{~min} ; 37$ cycles of denaturation at $94^{\circ} \mathrm{C}$ for $30 \mathrm{~s}$, annealing at $55^{\circ} \mathrm{C}$ for $30 \mathrm{~s}$, and extension at $75^{\circ} \mathrm{C}$ for $45 \mathrm{~s}$; and a final extension step at $72^{\circ} \mathrm{C}$ for $5 \mathrm{~min}$.

\section{Identification of PCR products}

The PCR products were detected by $2 \%$ agarose gel electrophoresis (Figure 1).

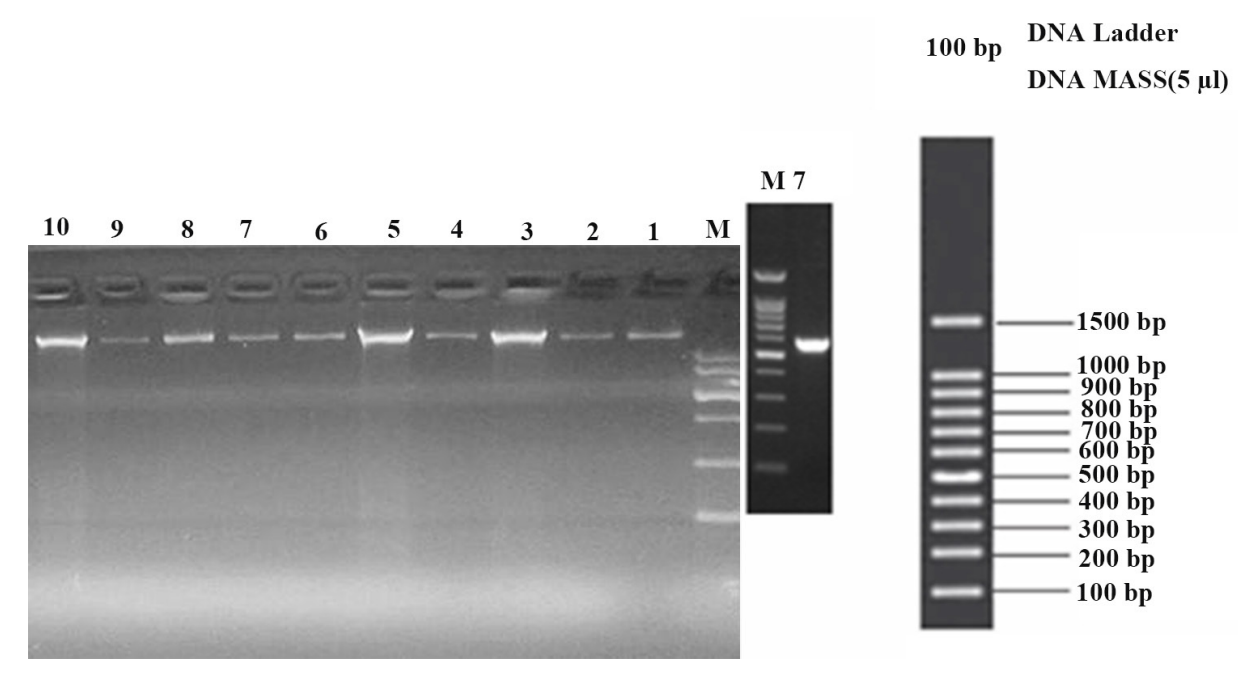

Figure 1. Agarose gel displaying the amplified TTR gene extracted from various members of a family with familial vitreous amyloidosis.

\section{Sequencing and analysis}

The products were sequenced using a DNA sequencer (3730XL) after purification. The primers were designed and synthesized by Invitrogen. The PCR products were sequenced using the fluorescence marker method, and the sequencing results were analyzed by DNAMAN (for Windows) v.5.2.2.0 and Chromas. The obtained sequence was aligned by comparing with the start codon anterior segment of the TTR gene available at the National Center for Biotechnology Information (NCBI). 


\section{RESULTS}

The results showed that the length of upstream regulation regions in the 10 subjects was in accordance with the length of the reference sequence extracted from GenBank. The sequence alignment revealed the absence of any mutations in 6 of the subjects (4 patients and 2 without disease), which corresponded with the sequencing results. The mutations in the subjects occurred at the same site $(-743, A \rightarrow T)$. (Figure 2 and 3$)$.
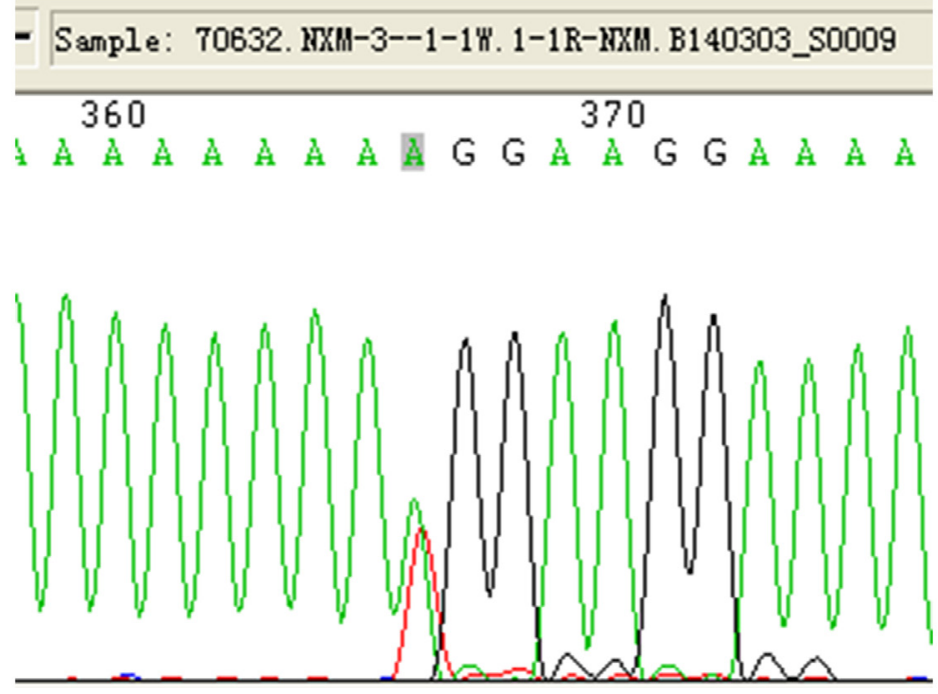

Figure 2. Results of sequencing of the upstream regulatory region of the TTR gene in study subjects $(-743, A \rightarrow T)$.

$\frac{\text { - Sample: T0632. KXM-10--1-1\%'. 1-1R-kXM. B140303_S0016 }}{\text { A A A A A A A A T G G A A G G A A A A }}$

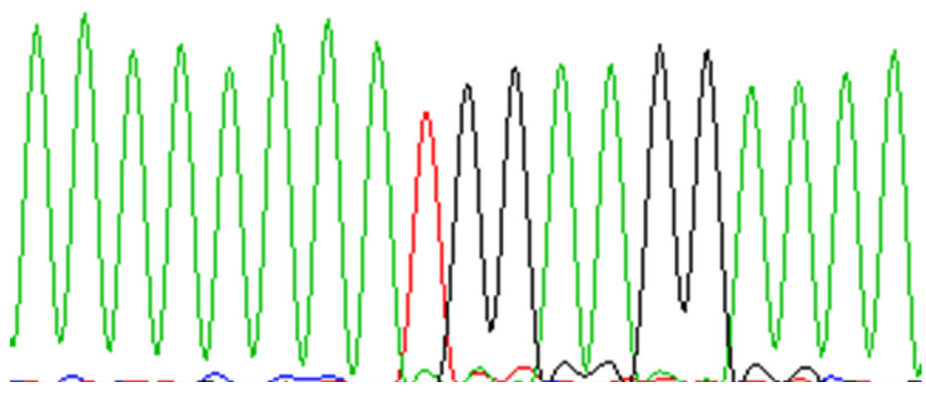

Figure 3. Sequencing results of the upstream regulatory region of the TTR gene in controls $(-743, A A)$. 


\section{DISCUSSION}

Changes to the TTR structure have been identified as the main factors inducing familial amyloidosis (Coelho et al., 2012). Domestic reports regarding vitreous amyloidosis in the eye, however, are very few. Familial vitreous amyloidosis was studied in a family from the AnShun region of the Gui Zhou Province, which confirmed that amyloid substances resulted in vitreous opacity. This familial mutation site, located in the third exon of the TTR gene, which induced a mutation in the 83rd amino acid (Gly83Arg), was found to be a heterozygous mutation (Xie et al., 2013).

During preparatory analyses, a point mutation (Gly83Arg) of the TTR gene was observed in 2 diseased family members. DNA mutation was also not observed in 2 members diagnosed with vitreous amyloidosis. We attempted to validate our theory that the abnormal base sequence or the level of modification in the upstream regulatory region of TTR gene could be pathogenic by regulating the non-mutated TTR mRNA and protein sequences.

Blood was collected from 10 members of a family with vitreous amyloidosis; the DNA sequencing results showed the presence of a base heterozygous mutation $(-743, A \rightarrow T)$ in 6 subjects: 4 patients and 2 without disease. Therefore, we believed that vitreous amyloidosis might be associated with a point mutation in the upstream regulatory region of the TTR gene.

The gene expression was regulated on multiple levels: the eukaryotic cells were regulated at the DNA and chromosomal, post-transcriptional, translational, post-translational, and mRNA degradation levels; moreover, the cis-acting elements and trans-acting factors, such as the promoter, enhancer, and terminator, were also regulated. Translational regulation was most important in the promoter region (Pedersen et al., 1999). Although promoters also influenced transcription, the site of mutation in the upstream regulatory region of the TTR gene was not in the typical TATA-box and GC-box in this study (Zhang and Qi, 2008; Kutach and Kadonaga, 2000).

A promoter prediction software was used to predict the promoter region in the TTR upstream regulatory region; the mutation site was found to be located far from the promoter or start codon (-743 bp), and not in promoter region. This basically indicated that changes in the promoter region did not induce corresponding changes in the expression of the regulatory region.

Future studies must attempt to determine the mechanism with which changes in the upstream regulatory region induce changes in the mRNA and protein expression, and identify any direct relationship or causality between these changes and base changes in the upstream region. Moreover, we must attempt to establish if the regulation of gene expression induced changes in the regulatory region or if the opposite holds true.

\section{Conflicts of interest}

The authors declare no conflict of interest.

\section{ACKNOWLEDGMENTS}

Research supported by the National Science Foundation.

\section{REFERENCES}

Coelho T, Maia LF, Martins da Silva A, Waddington Cruz M, et al. (2012). Tafamidis for transthyretin familial amyloid polyneuropathy: a randomized, controlled trial. Neurology 79: 785-792.http://dx.doi.org/10.1212/WNL.0b013e3182661eb1 
Ikeda S, Nakazato M, Ando Y and Sobue G (2002). Familial transthyretin-type amyloid polyneuropathy in Japan: clinical and genetic heterogeneity. Neurology 58: 1001-1007.http://dx.doi.org/10.1212/WNL.58.7.1001

Kutach AK and Kadonaga JT (2000). The downstream promoter element DPE appears to be as widely used as the TATA box in Drosophila core promoters. Mol. Cell. Biol. 20: 4754-4764.http://dx.doi.org/10.1128/MCB.20.13.4754-4764.2000

Pedersen AG, Baldi P, Chauvin Y and Brunak S (1999). The biology of eukaryotic promoter prediction--a review. Comput. Chem. 23: 191-207.http://dx.doi.org/10.1016/S0097-8485(99)00015-7

Westermark P, Benson MD, Buxbaum JN, Cohen AS, et al. (2002). Amyloid fibril protein nomenclature -- 2002. Amyloid 9: 197-200.http://dx.doi.org/10.3109/13506120209114823

Xie B, Cai SJ and Li H (2013). Gly83Arg mutation of thyroid hormone binding proteins in family vitreous amyloidosis. Zhonghuayandibingzazhi 29: 569-571.

Zhang XH and Qi YX (2008). Analysis of TATA-box, GC-box and CAAT-box in eukaryotic promoter. J. Anhui Agri. Sci. 36: 1380-1381. 